\title{
ANL/PHY/CP--84488
}

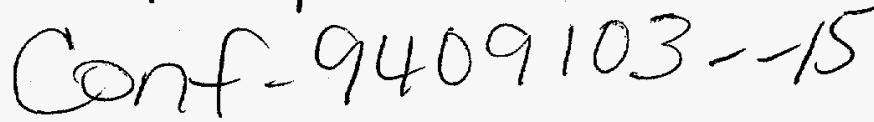

\section{Prospects for HERMES-Spin Structure Studies at HERA}

\author{
H. E. Jackson \\ Physics Division, Argonne National Laboratory, Argonne, IL 60439-4843
}

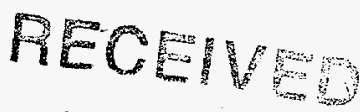

JAN $2>1995$

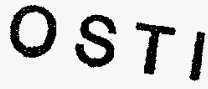

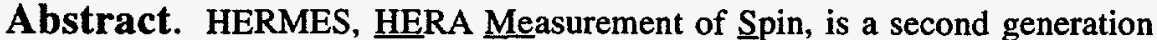
experiment to study the spin structure of the nucleon by using polarized internal gas targets in the HERA 35-GeV electron storage ring. Scattered electrons and coincident hadrons will be detected in an open geometry spectrometer which will include particle identification. Measurements are planned for each of the inclusive structure functions, $g_{1},(x), g_{2}(x), b_{1}(x)$ and $A(x)$, as well as the study of semi-inclusive pion and kaon asymmetries. Targets of hydrogen, deuterium and ${ }^{3} \mathrm{He}$ will be studied. The accuracy of data for the inclusive structure functions will equal or exceed that of current experiments. The semi-inclusive asymmetries will provide a unique and sensitive probe of the flavor dependence of quark helicity distributions and properties of the quark sea. Monte Carlo simulations of HERMES data for experiment asymmetries and polarized structure functions are discussed.
\end{abstract}

\section{INTRODUCTION}

Recent studies of the spin structure functions of the nucleon have yield very interesting, if unexpected results, quite different from naive expectations. The data from these experiments $(1,2)$ has been interpreted as showing that the quarks in the nucleon carry only $\sim 1 / 3$ of the spin of the nucleon, i.e. about $50 \%$ of the value which results from a careful full relativistic treatment (3) of the nucleon spin structure. These data also violate at the 3- $\sigma$ level the Elles-Jaffe sum rule (4) for $\int_{0}^{I} d x g_{1} p(n)(x)$, and suggest that the strange sea in the nucleon is polarized. A large body of theoretical speculation on the spin structure of the nucleon, based on the data from these experiments, has developed. It is evident that detailed and comprehensive experiments will be necessary to provide a precise description of the spin structure of the nucleon. HERMES, a study of spin structure at HERA, is such an experiment. The participants and collaborating institutions are listed in Appendix A. Spin dependent deep inelastic scattering from the proton and the neutron will be measured by using the longitudinally polarized electron beam of the HERA storage ring at beam energies in excess of $27.5 \mathrm{GeV}$. Polarized internal gas targets of hydrogen, deuterium, and ${ }^{3} \mathrm{He}$ will scatter the circulating electron beam of which is polarized to a level greater than $50 \%$ by the Sokolov-Ternov effect (5). Scattered electrons and associated hadrons will be detected in a large acceptance open geometry spectrometer. The $x$ dependence of the proton and neutron spindependent structure functions will be measured to high precision. The determination of the integral over $x$ will test the Bjorken Sum rule to high precision. In addition, in HERMES measurements will be possible of leading hadrons from 


\section{DISCLAIMER}

This report was prepared as an account of work sponsored by an agency of the United States Government. Neither the United States Government nor any agency thereof, nor any of their employees, makes any warranty, express or implied, or assumes any legal liability or responsibility for the accuracy, completeness, or usefulness of any information, apparatus, product, or process disclosed, or represents that its use would not infringe privately owned rights. Reference herein to any specific commercial product, process, or service by trade name, trademark, manufacturer, or otherwise does not necessarily constitute or imply its endorsement, recommendation, or favoring by the United States Government or any agency thereof. The views and opinions of authors expressed herein do not necessarily state or reflect those of the United States Government or any agency thereof. 


\section{DISCLAIMER}

Portions of this document may be illegible in electronic image products. Images are produced from the best available original document. 
spin-dependent deep inelastic scattering. This will provide important additional information on the contribution of the valence and sea quarks to the spin asymmetries. HERMES will provide a new experimental frontier in deep inelastic scattering and an unprecedented capability to study spin-dependent effects in electron scattering from the nucleon and the few-nucleon systems.

\section{PHYSICS OF SPIN ASYMMETRIES}

The basic features of the spin structure of the nucleon are characterized by the spin structure functions $g_{1}(x)$ and $g_{2}(x)$. Experimentally, these structure functions describe the difference in the deep-inelastic electron scattering cross sections for opposite target polarizations according to the equation

$$
\begin{gathered}
\frac{d^{3}(\sigma(\alpha)-\sigma(\alpha+\pi))}{d x d y d \sigma}=\frac{e^{4}}{4 \pi^{2} Q^{2}}\left[\cos \alpha\left(\left[1-\frac{y}{2}-\frac{y^{2}}{4} \gamma^{2}\right] g_{1}\left(x, Q^{2}\right)-\frac{y}{2} \gamma^{2} g_{2}\left(x, Q^{2}\right)\right)\right. \\
\left.-\sin \alpha \cos \phi \sqrt{\gamma^{2}\left(1-y-\frac{y^{2}}{4} \gamma^{2}\right)}\left(\frac{y}{2} g_{1}\left(x, Q^{2}\right)+g_{2}\left(x, Q^{2}\right)\right)\right]
\end{gathered}
$$

where, as indicated in Fig. 1, $\alpha$ is the angle between the beam momentum and the target polarization, $\phi$ is the angle between the plane defined by the beam direction and target polarization, and the scattering plane defined by the directions of the incident and scattered lepton. $Q^{2}$ is the negative square of the invariant mass of the virtual photon, $x=Q^{2} / 2 M v$ and $y=v / E$ are the Bjorken scaling variables, $E$ and $E^{\prime}$ are the energies of the incident and the scattered lepton, and $M$ is the nucleon mass. The quantity $\gamma$ is defined by $\gamma=\sqrt{Q^{2} / v}$. The structure functions can be extracted from the polarization asymmetry measured for parallel and anti-parallel target-beam spins, $A_{||}(x)$ and the transverse asymmetry, $A \perp(x)$, which corresponds to target polarization perpendicular to the beam. These measured asymmetries are related to the virtual photon asymmetries, $A_{1}=\left(\sigma_{1 / 2}-\right.$ $\left.\sigma_{3 / 2}\right) /\left(\sigma_{1 / 2}+\sigma_{3 / 2}\right)$ and $A_{2}=\sigma_{T L} / \sigma_{T}$, by the relations

$$
\begin{aligned}
& A_{\|}=D \cdot\left(A_{1}+\eta \cdot A_{2}\right) \\
& A_{\perp}=d \cdot\left(A_{2}-\xi \cdot A_{1}\right) .
\end{aligned}
$$

where the kinematic factors are defined by

$$
\begin{gathered}
D=\frac{y(2-y)}{y^{2}+2(1-y)(1+R)} \\
\eta=\frac{2 \gamma(1-y)}{(2-y)}
\end{gathered}
$$




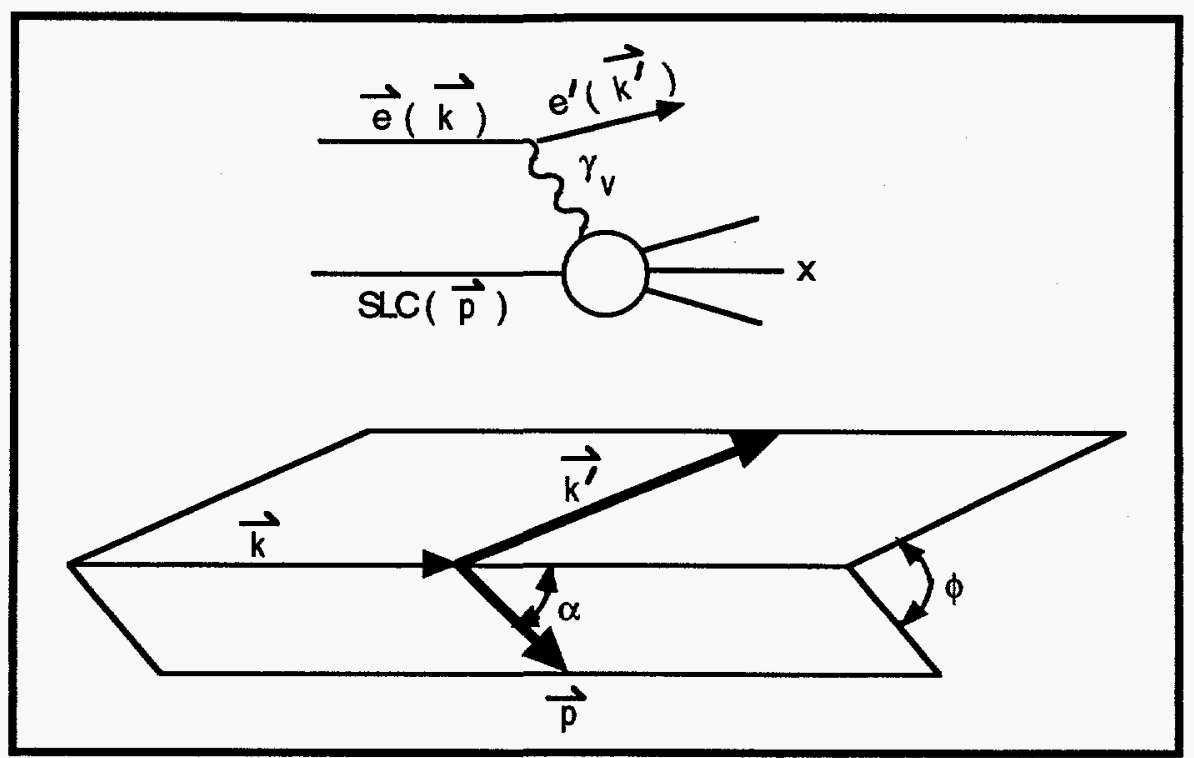

FIGURE 1. Kinematics for polarized deep-inelastic electron scattering.

$$
\begin{aligned}
& d=D \sqrt{\frac{2 \varepsilon}{1+\varepsilon}} \\
& \xi=\eta \frac{1+\varepsilon}{2 \varepsilon}
\end{aligned}
$$

with $\varepsilon=(1-y) /\left(1-y+y^{2} / 2\right)$ being the degree of transverse polarization of the virtual photon. The asymmetries $A_{1}$ and $A_{2}$ can be expressed in terms of $g_{1}$ and $g_{2}$ as

$$
\begin{aligned}
& A_{1}=\frac{g_{1}-\gamma^{2} g_{2}}{F_{1}} ; \\
& A_{2}=\frac{\gamma\left(g_{1}+g_{2}\right)}{F_{1}}
\end{aligned}
$$

Since $\gamma, \eta$, and $R$ are usually small, it is customary to neglect the contribution of $A_{2}$ to the $A_{\| l}$ and $A_{I}$ to $A_{p e r}$ with the result that to good approximation

$$
A_{\|} \approx \frac{g_{1}}{F_{1}} \cdot D \quad A_{\perp}=\frac{\gamma\left(g_{1}+g_{2}\right)}{F_{1}} \cdot d
$$

The experimentally measured electron asymmetries include the effects of beam and target polarizations, $P_{b}$ and $\mathrm{P}_{\mathrm{t}}$, as well as any target dilution, $f$, i.e. $A_{\text {exp }}=$ 
$P_{b} P_{t} A_{e}$. In the Quark-Parton Model $g_{1}(x)=1 / 2 \sum e_{i}{ }^{2}\left(q_{i}^{\uparrow}(x)-q_{i}^{\downarrow}(x)\right)$, so that the net helicity distribution summed over quark flavor can be estimated from $A_{\|}$with the aid of the known unpolarized structure function, $F_{I}(x)$. The integral of $g_{I}(x)$ is of great importance (3) since it provides an independent combination of the net helicities, $\Delta u, \Delta d$, and $\Delta s$ in the proton. The value of this integral, when combined with data or neutron and hyperon $\beta$-decay, can be used to infer values of $\Delta u, \Delta d$, and $\Delta s$. This is the treatment which has been used in previous experiments to conclude that the spin carried by the quarks in the nucleon is anomolously small. In HERMES it will be possible to measure $A_{2}$ with sufficient precision to avoid the approximation made in Eq. (9).

Because of the ability to identify hadrons, HERMES will be able to provide unambiguous identification of pions and later kaons and their associated spin asymmetries. These data can provide important information on the flavor dependence of quark helicity distributions in the nucleon, and be useful in separating the contributions of the valence and sea quarks to the total nucleon polarization. For polarized pion production, the Quark-Parton model gives (16) up to a constant factor

$$
\begin{gathered}
N_{\uparrow \downarrow}^{\pi^{+} \sim} \frac{4}{9} u_{+}(x) D_{u}^{\pi^{+}}(z)+\frac{1}{9} d_{+}(x) D_{d}^{\pi^{+}}(z)+\frac{1}{9} d_{+}(x) D_{d}^{\pi^{+}}(z) \\
+\frac{1}{9} S_{+}(x) D_{s}^{\pi^{+}}(z)+\frac{1}{9} \vec{S}_{+}(x) D_{s}^{\pi^{+}} .
\end{gathered}
$$

where $q_{+}\left(q^{-}\right)$is the distribution function of a quark $q$ with helicity parallel (antiparallel) to the proton helicity and $D_{q}^{h}(z)$ is the fragmentation function of a quark into a hadron $h$ with energy $E^{h}=z \cdot v$, with similar form for other pion charges and helicities. Factorization and helicity independence of the fragmentation function is assumed. An example of a useful hadron asymmetry is that of the net pion charge

$$
A_{R}^{\pi}(x)=\frac{N_{\uparrow}^{\pi^{+}-\pi^{-}}(x)-N_{\downarrow}^{\pi^{+}-\pi^{-}}(x)}{N_{\downarrow}^{\pi^{+}-\pi^{-}}(x)+N_{\downarrow}^{\pi^{+}-\pi^{-}}(x)}
$$

This quantity should be independent of sea quark effects, with the result that measurements of this quantity for the proton and the deuteron can be used to deduce $\Delta u_{v}(x)$ and $\Delta d_{v}(x)$ in the proton.

\section{EXPERIMENTAL DETAILS}

The internal polarized gas target for HERMES is shown schematically in Fig. 2. It consists of an open-ended thin walled storage cell through which the circulating electron beam of the HERA accelerator passes. The cell is filled with polarized protons, deuterons, or ${ }^{3} \mathrm{He}$ from an atomic beam source as shown or from an 


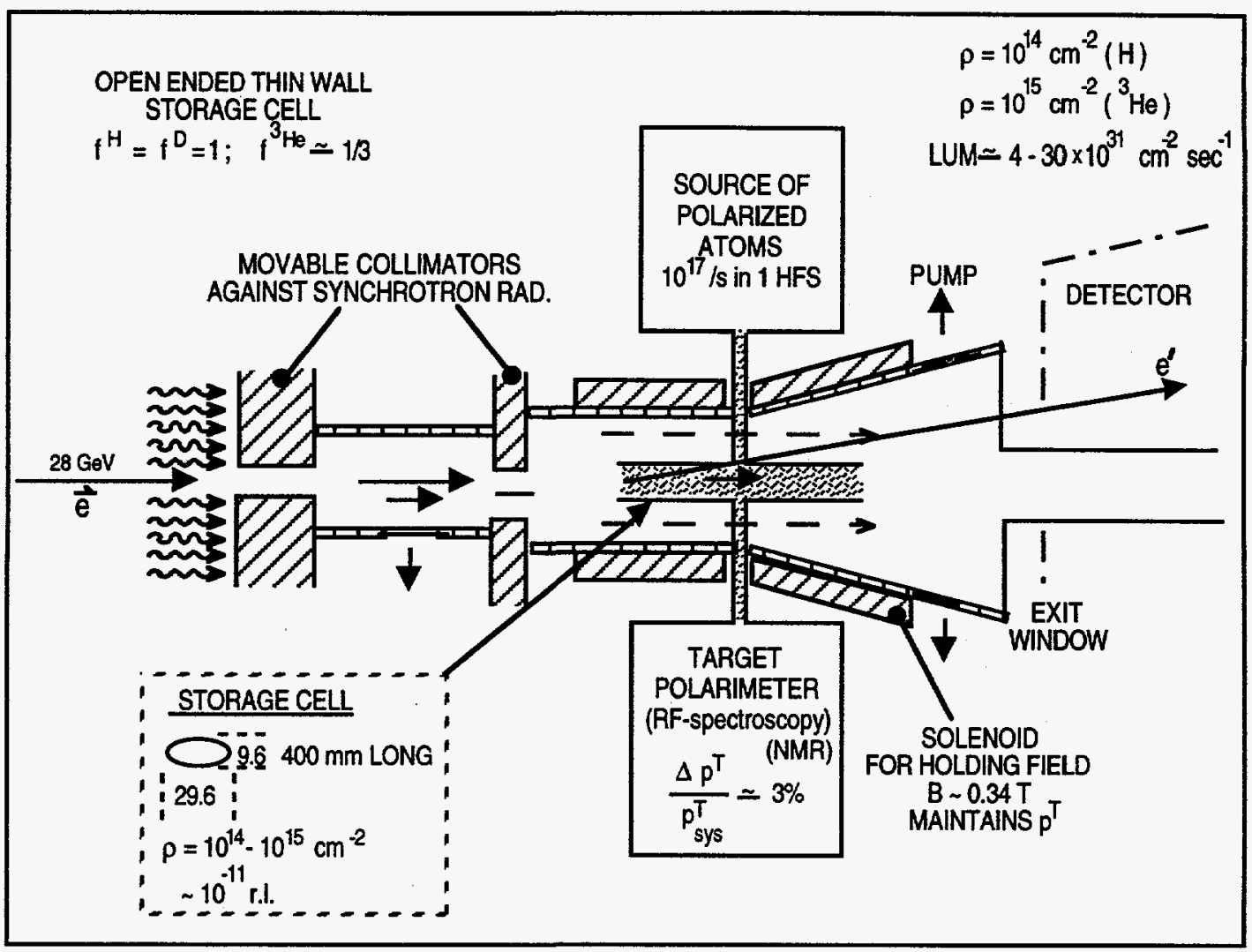

FIGURE 2. Schematic view of HERMES internal polarized gas target.

optically pumped ${ }^{3} \mathrm{He}$ source. A solenoidal holding field provides a quantization axia for the target polarization. The target densities will be about $10^{14}$ atoms $/ \mathrm{cm}^{2}$ for hydrogen and deuterium and $10^{15}$ atoms $/ \mathrm{cm}^{2}$ for ${ }^{3} \mathrm{He}$. Luminosities will be in the range of 4-30 $10^{31}$ nucleons $\mathrm{cm}^{-2} \mathrm{sec}^{-1}$. The gases are pure so that there is no target dilution. The extremely thin target density minimizes internal radiative corrections.

The experiment will be located in the east Hall of the HERA accelerator with the interaction area reconfigured so that the proton beam bypasses the HERMES target. The beam is polarized transverse to the beam direction by the Sokolov-Ternov effect. In a perfect machine it will reach $92 \%$. Spin rotators located at the entrance and exit of the hall (7) will precess the spin direction from vertical to longitudinal at the target position and following the target back to the vertical position. The polarization of the beam with the rotators in place has been measured for both positrons and electrons. The results of a typical measurement are shown in Fig. 3. Longitudinal polarizations of about $60 \%$ are stable and reproducible. The polarization time is about 20 minutes. As a result of the ease with which the direction of the target polarization can be changed, it will be possible to study parallel and perpendicular polarization asymmetries for all targets. In addition to measurements of $g_{1}$ and $g_{2}$, and the Bjorken sum rule, estimates will be made of 


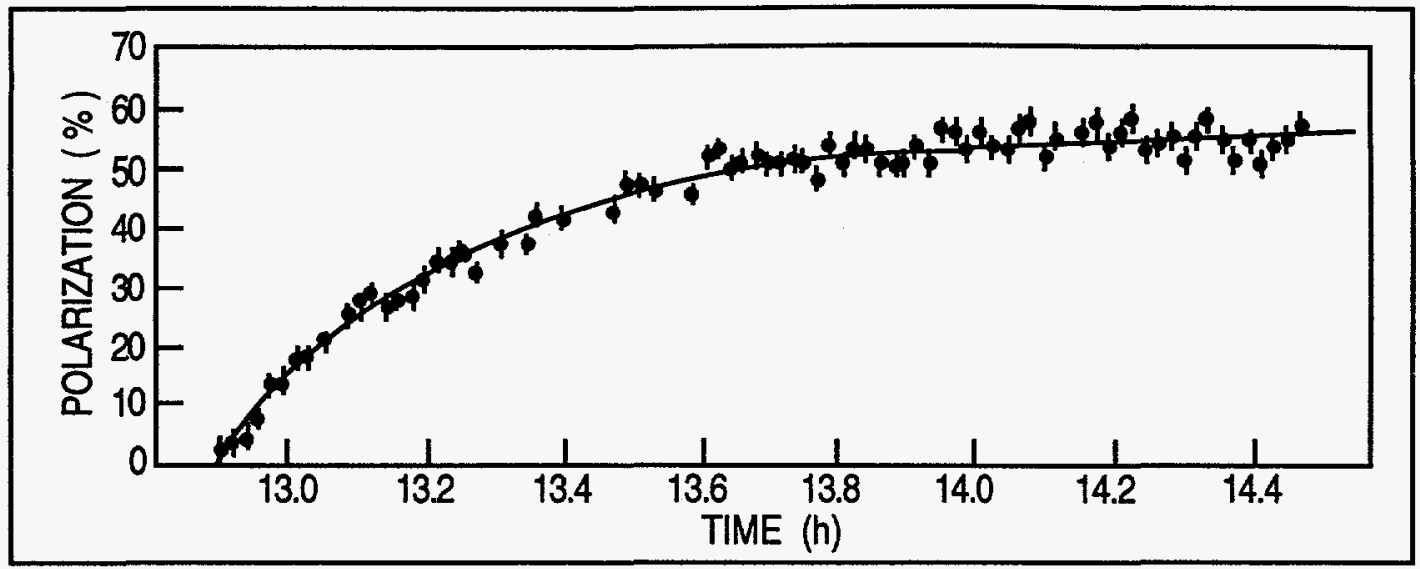

FIGURE 3. Rise of electron polarization at HERA with spin rotations in place. Polarization is longitudinal at the HERMES target.

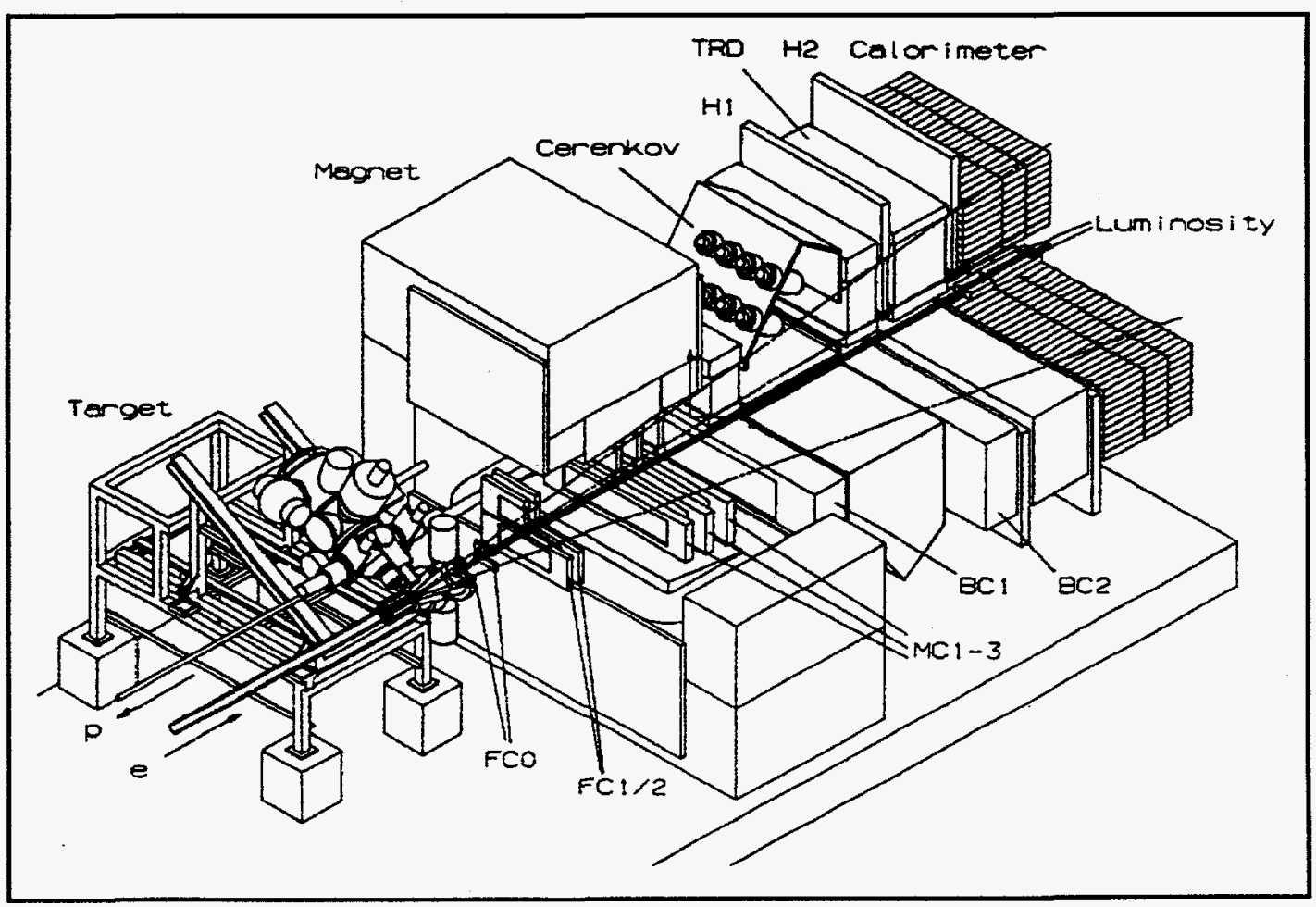

FIGURE 4. Schematic view of the HERMES magnetic spectrometer. 
TABLE 1. Projected sum rule uncertainties

\begin{tabular}{ccc} 
Target & Polarization & Quantity to be measured \\
\hline$H$ & $\rightarrow$ & $g_{1}^{p}(x)$ \\
$D$ & $\rightarrow$ & $g_{1}^{p}(x), g_{1}^{n}(x), \int\left(g_{1}^{p}-g_{1}^{n}\right) d x, b_{1}(x)$ \\
$3 H e$ & $\rightarrow$ & $g_{1}^{n}(x), \int\left(g_{1}^{p}-g_{1}^{n}\right) d x$ \\
$H$ & $\perp$ & $g_{2}^{p}(x)$ \\
$D$ & $\perp$ & $g_{2}^{p}(x), \Delta(x)$ \\
$3 H e$ & $\perp$ & $g_{2}^{n}(x)$
\end{tabular}

the higher order structure functions $b_{1}$ (9) and $\Delta$ (8) which are relevant to the deuteron. A summary of the measurements planned is shown in Table 1.

The HERMES spectrometer, shown in Fig. 4, is an open geometry system. A magnet with a bending strength of $1.3 \mathrm{~T}-\mathrm{m}$ will be used to measure particle momenta. The magnet is divided into 2 symmetric parts by a horizontal flux plate through which both the electron and proton beam pass. A fly's eye $\mathrm{Pb}$ glass calorimeter will provide a level one trigger. A transition radiation detector will provide strong discrimination against pions. Tracking chambers before, in the magnetic field, and behind the magnet will be used to measure particle trajectories and momenta. A system of segmented hodoscopes with road structure will be part of the level one trigger. For detection of hadrons in coincidence with the scattered lepton, a pair of threshold gas Cerenkov counters will be used. The radiator, tetrafluoromethane, will have Cerenkov light thresholds of $4.4 \mathrm{GeV}$ for pions, 15.8 $\mathrm{GeV}$ for kaons, and $30.0 \mathrm{GeV}$ for protons. Each unit will be divided into 20 cells viewed by individual photomultipliers. The kinematic region which will be covered by the spectrometer for a beam energy of $28 \mathrm{GeV}$ is shown in Fig. 5. A minimum momentum transfer of $1(\mathrm{GeV} / \mathrm{c})^{2}$ will allow interpretation of the data in terms of the quark-parton model. The invariant mass cut of $\mathrm{W}>2 \mathrm{GeV}$ will eliminate the resonance region. A cut on the energy loss at $y<0.85$ will eliminate the region of large radiative corrections. The spectrometer angular acceptance will range from 40 $\mathrm{mr}$ to $220 \mathrm{mr}$.

\section{PROJECTED PERFORMANCE}

The precision of measurements of structure functions, statistical plus systematic errors in HERMES, should be at least as good or perhaps somewhat improved than any of other experiments in progress or planned. This point is illustrated by Fig. 6 where recent results for $g_{l}^{n}$ are plotted together with projected data for a 600 hour run in HERMES at the expected luminosity. Projected uncertainties in the measured 


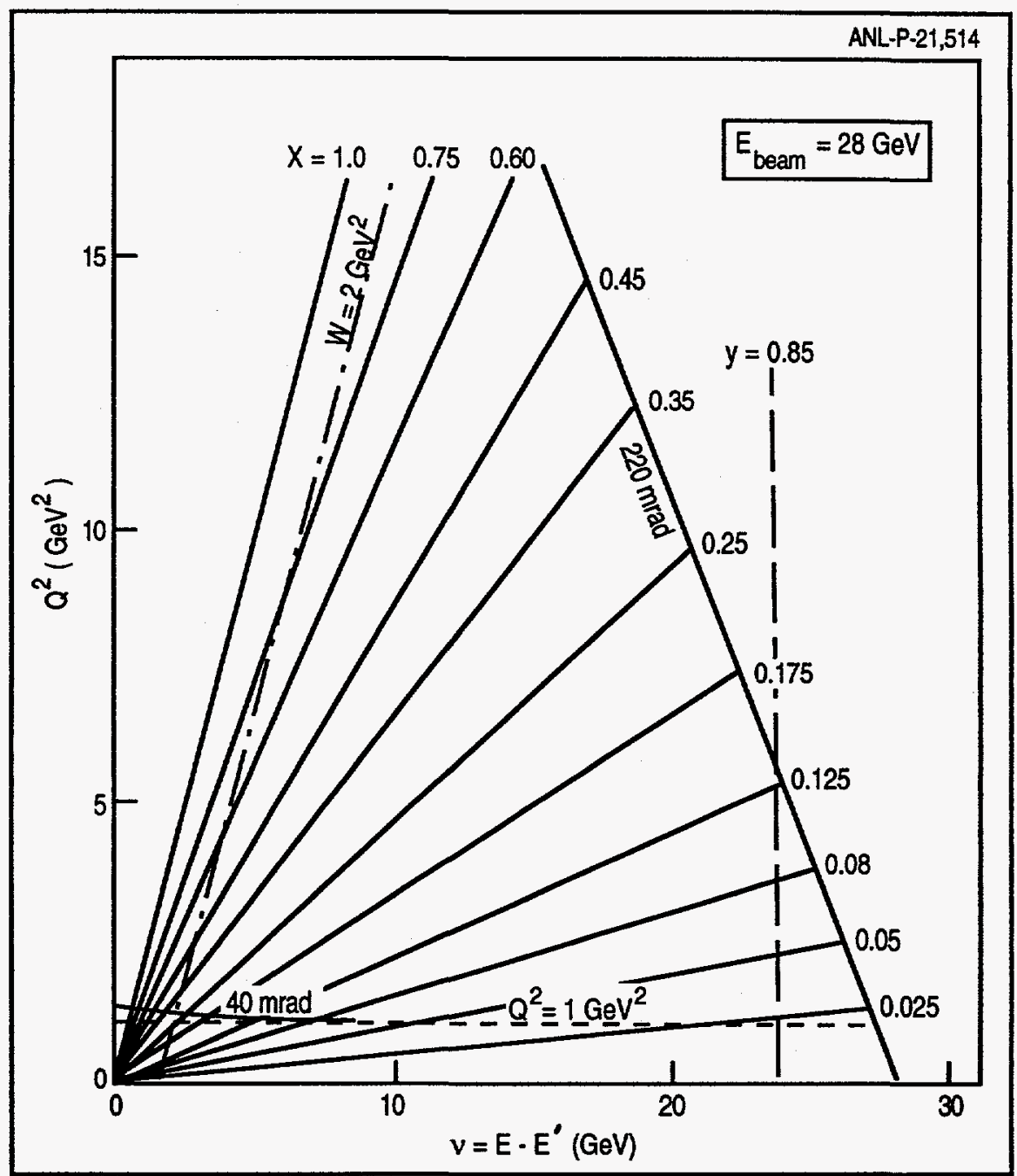

FIGURE 5. Kinematic plane in HERMES for an electron beam energy of $28 \mathrm{GeV}$.

values of $\int g_{1}^{p}$ and $\int g_{1}^{n}$ as well as the Bjorken sum rule are presented in Table 2. HERMES should be competitive with the best experiments.

A knowledge of the structure function $g_{2}(x)$ is of interest theoretically as a measure of higher twist effects and because its value is required to make an unambiguous determination of $g_{1}(x)$ from the longitudinal asymmetry. It will be estimated in HERMES from measurements of $A_{2}(x)$ in a target transverse asymmetry (see Eq. (3)). In Fig. 7 the statistical uncertainties are shown for the extracted $g_{2}^{p}(x)$ together with curves for the leading twist contribution and a twist-3 contribution in a specific model. Also shown is the positivity limit for $A_{2}=\sqrt{R}$. The data will constrain the $x$-dependence of $g_{2}(x)$ rather well. This will also provide a test of the Wandzura-Wilsek relation (11) and possibly a separation of the twist-3 contribution arising from quark-gluon correlations. 


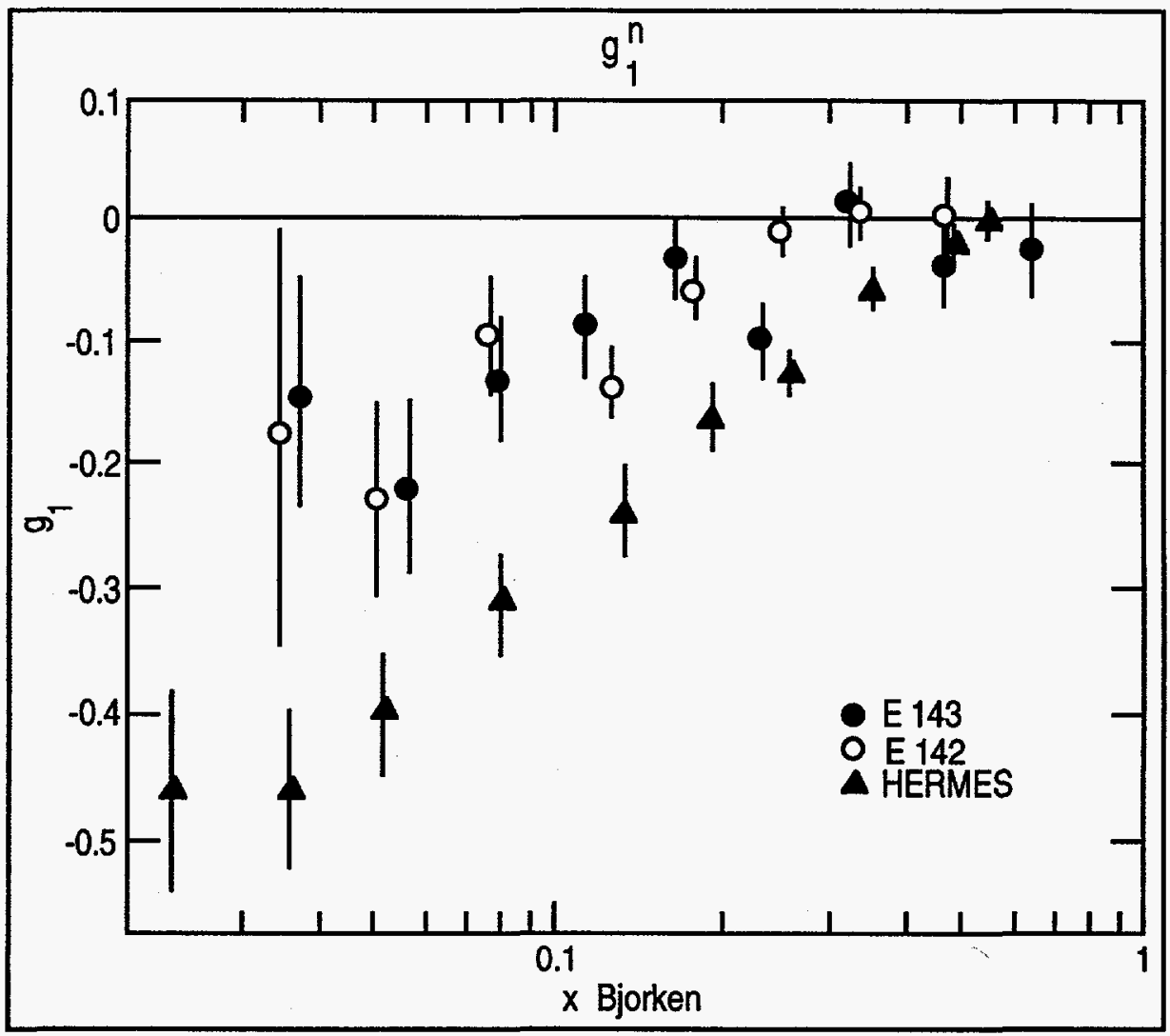

FIGURE 6. The neutron spin structure function $g_{1}^{n}(x)$ for a 300 -hour run in HERMES. Also shown are data from recent measurements at SLAC (see Ref. (2)).

In the case of the deuteron, which is a spin-1 target, there are two new structure functions, $b_{1}(x)$ and $\Delta(x)$ which describe the additional physics of the spin one system. $b_{1}(x)$ has a structure similar to a tensor polarization and can be measured in HERMES by scattering an unpolarized beam from a deuterium target arranged in each of the three possible substates $m_{I}=+1,0,-1 . \Delta(x)$ is measured by scattering an unpolarized electron beam from a polarized deuterium target in the $m_{I}=0$ state with the target polarization perpendicular the beam direction and measuring the $\phi$ distribution of the scattered electrons. It is sensitive to the gluon components in the deuteron.

\section{SEMI-INCLUSIVE ASYMMETRIES}

With particle identification, HERMES will be able to provide unambiguous identification of pions, and later kaons, and measurements of their associated asymmetries. These data can provide important information on the flavor dependence of quark helicity distributions in the nucleon, and be useful in separating the contributions of the valence and sea quarks to the total quark 
TABLE 2. Projected Sum Rule Uncertainties. Values for the Bjorken Sum Rule $\int_{0}^{l}\left[g_{I}^{p}(x)-g_{I}^{n}(x)\right] d x=\frac{1}{6}\left(\frac{g_{A}}{g_{V}}\right)\left[1+o\left(\alpha_{s}\right)\right]$ are tabulated in the last column.

\begin{tabular}{|c|c|c|c|}
\hline & $\int g_{1}^{p}$ & $\int g_{l}^{n}$ & $\int g_{1}^{p}-g_{1}^{n}$ \\
\hline $\begin{array}{l}\text { Measured } \\
\text { Value }\end{array}$ & 0.13 & -0.03 & 0.15 \\
\hline $\begin{array}{l}E 142 \\
\left({ }^{3} \mathrm{He}\right)\end{array}$ & & $\begin{array}{c} \pm 0.011 \\
(36 \%)\end{array}$ & $\begin{array}{c} \pm 0.016 \\
(10 \%)\end{array}$ \\
\hline $\begin{array}{c}E{ }_{143} \\
\left(\mathrm{NH}_{3}, \mathrm{NO}_{3}\right)\end{array}$ & $\begin{array}{c} \pm 0.011 \\
(8 \%)\end{array}$ & $\begin{array}{c} \pm 0.014 \\
(46 \%)\end{array}$ & $\begin{array}{c} \pm 0.025 \\
(17 \%)\end{array}$ \\
\hline $\begin{array}{c}\mathrm{SMC} \\
\left(\mathrm{C}_{4} \mathrm{H}_{9} \mathrm{OH},\right. \\
\left.\mathrm{C}_{4} \mathrm{D}_{9} \mathrm{OD}\right)\end{array}$ & $\begin{array}{c} \pm 0.016 \\
(12 \%)\end{array}$ & $\begin{array}{l} \pm 0.043 \\
(143 \%)\end{array}$ & $\begin{array}{c} \pm 0.051 \\
(34 \%)\end{array}$ \\
\hline $\begin{array}{l}\text { HERMES } \\
(H, D)\end{array}$ & $\begin{array}{c} \pm 0.007 \\
(5 \%)\end{array}$ & $\begin{array}{c} \pm 0.009 \\
(30 \%)\end{array}$ & $\begin{array}{c} \pm 0.015 \\
(10 \%)\end{array}$ \\
\hline $\begin{array}{c}\text { HERMES } \\
(\mathrm{H}, 3 \mathrm{He})\end{array}$ & & $\begin{array}{c} \pm 0.007 \\
(23 \%)\end{array}$ & $\begin{array}{c} \pm 0.010 \\
(7 \%)\end{array}$ \\
\hline
\end{tabular}

polarization. Frankfurt and coworkers have calculated the asymmetry in the net pion charge, Eq. (11), for the proton and the deuteron

$$
\begin{aligned}
& A_{p}^{\pi}(x)=\frac{4 \Delta u^{v}(x)-\Delta d^{v}(x)}{4 u^{v}(x)-d^{v}(x)} \\
& A_{d}^{\pi}(x)=\frac{\Delta u^{v}(x)-\Delta d^{v}(x)}{u^{v}(x)-d^{v}(x)}
\end{aligned}
$$

From these two symmetries one can deduce both $\Delta u_{v}(x)$ and $\Delta d_{v}(x)$. They also note that one can use these results together with the data for the Bjorken sum rule to estimate the isospin symmetry of the polarization of the sea through the relationship

$$
6 \int_{0}^{l}\left[g_{l}^{p}(x)-g_{l}^{n}(x)\right] d x=\int_{0}^{l}\left[\Delta u^{v}(x)-\Delta d^{v}(x)+2(\Delta \bar{u}(x))\right] d x
$$




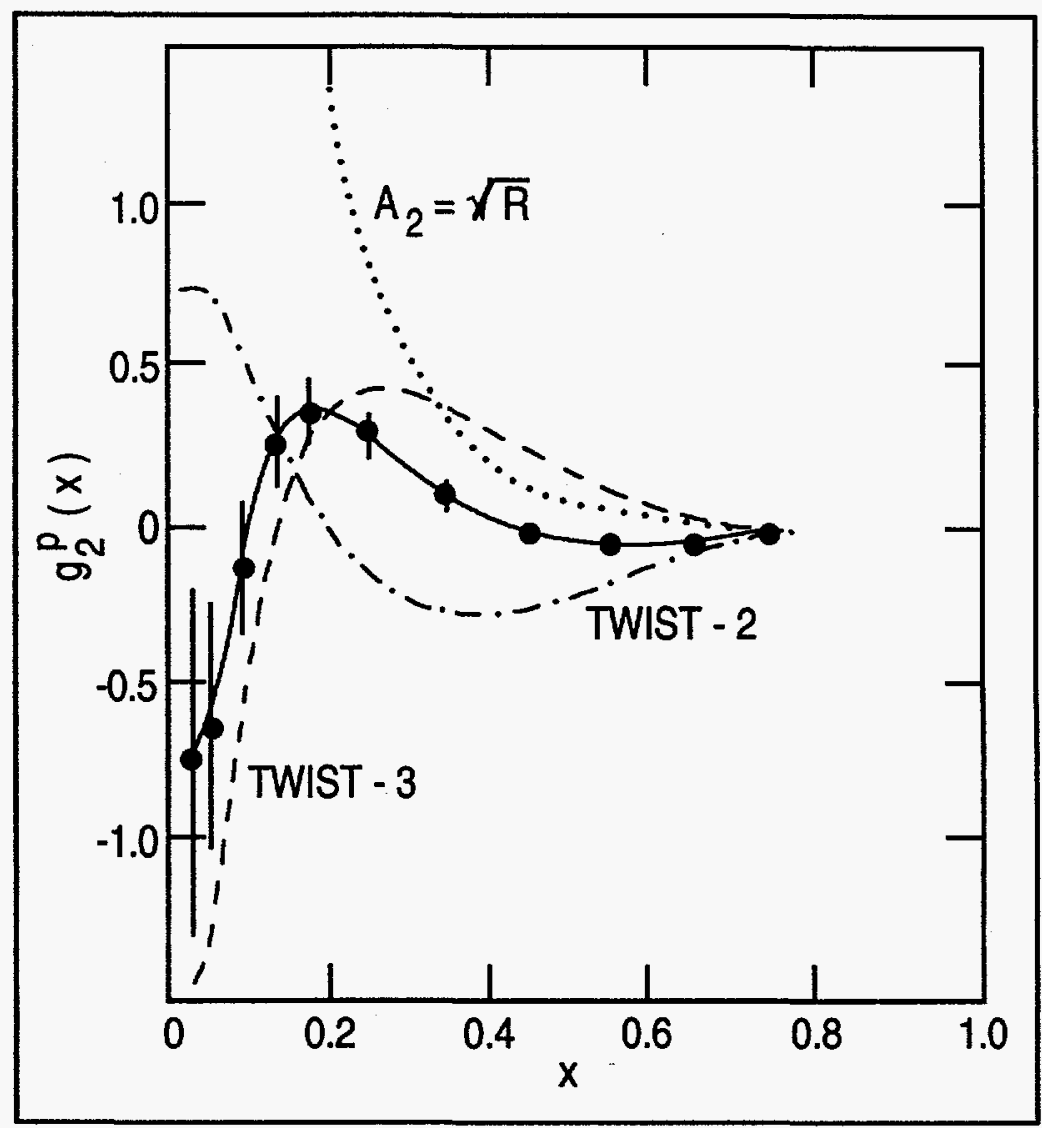

FIGURE 7. $g_{2}^{p}(x)$ as projected for an 800 -hour run by HERMES.

Monte Carlo simulations of these asymmetries have been carried out by Veltri et al. (13) Their results are presented in Fig. 8. The curve of Ross and Roberts (14) describes the result expected for a model with a polarized sea and glue. The dashed curve corresponds to a parton distribution due to Schäfer (15) in which the sea is unpolarized. The data corresponds to a standard HERMES run. In the region below $x=0.3$ one should be able to distinguish between the two models. The integrals of $\Delta u_{v}(x)$ and $\Delta d_{v}(x)$ can be estimated from this data to about 15-20\%.

After the first year of operation the HERMES threshold Cerenkov counters will be converted to Ring-imaging Cerenkov counters (RICH's). The current design is based on a $C_{4} F_{10}$ gas radiator. The projected ring separation which has been calculated for the converted system is shown in Fig. 9. The system will provide good separation of pions, kaons, and protons above $8 \mathrm{GeV}$ up to the highest hadron energies of the experiment. $K^{-}$asymmetries will be of particular interest because of the "all sea su " character of the $K^{-}$. For a proton target, the asymmetry in $K^{-}$production 


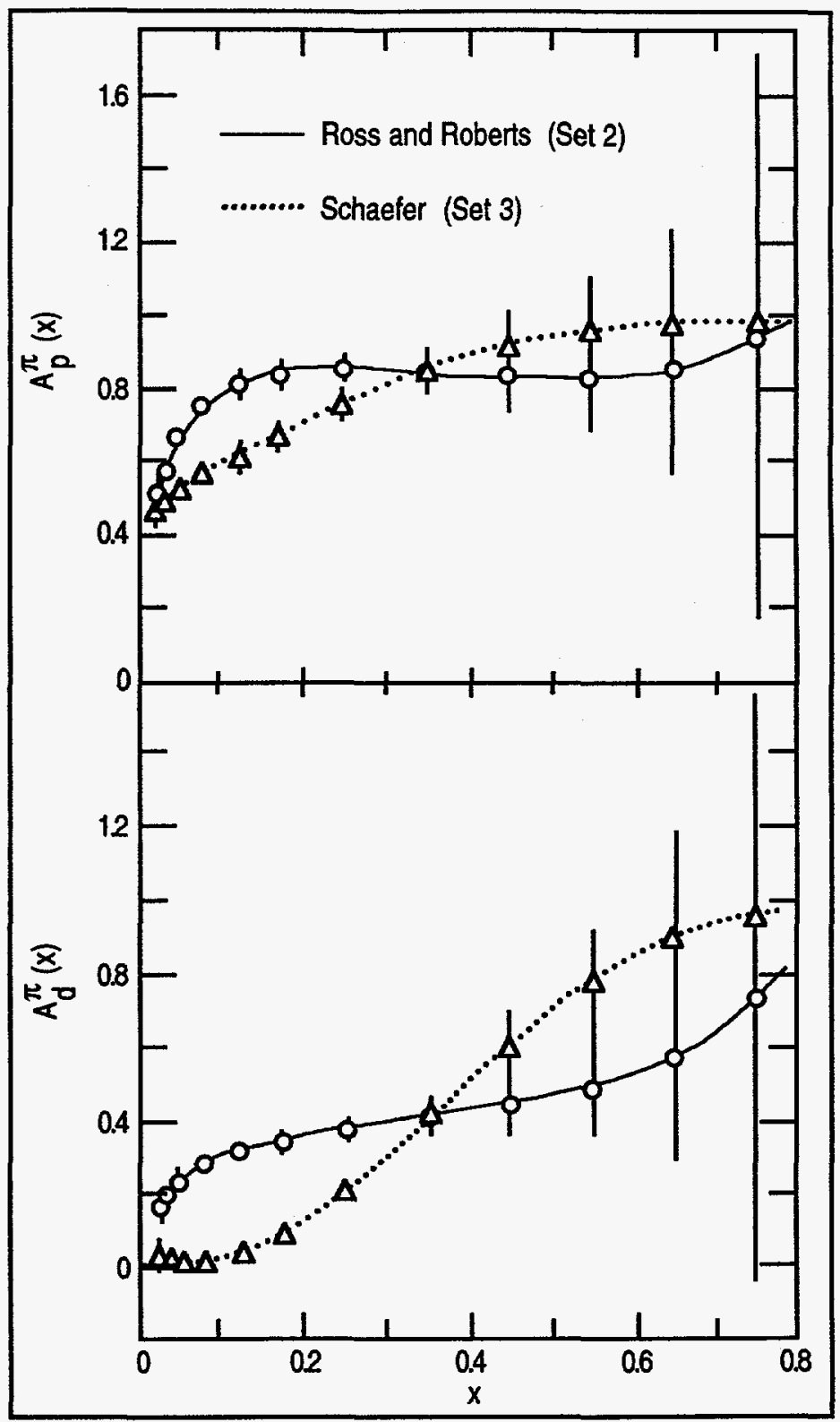

FIGURE 8. $A_{\mathrm{p}}^{\pi}$ and $A_{d}^{\pi}$ for two sets of parton distributions used in the HERMES Monte Carlo simulation. The error bars indicate the projected statistical accuracy for a 800-hour run.

$$
A_{p}^{K^{-}}=\frac{K_{\uparrow \downarrow}^{-}-K_{\downarrow \downarrow}^{-}}{K_{\uparrow \downarrow}^{-}+K_{\downarrow \downarrow}^{-}}
$$




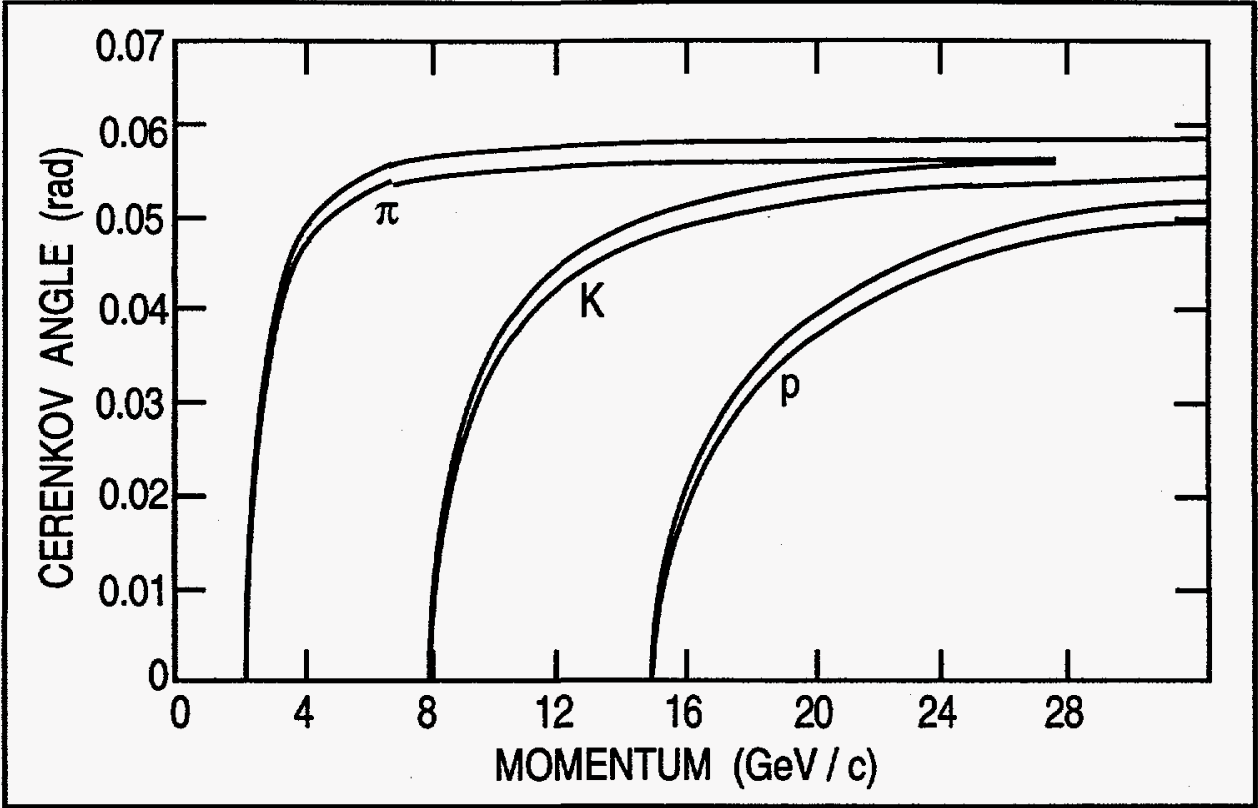

FIGURE 9. Anticipated $3 \sigma \pi / \mathrm{K} / \mathrm{p}$ separation with a RICH counter using a $1 \mathrm{~m}$ long $\mathrm{C}_{4} \mathrm{~F}_{10}$ radiator. The uncertainty in the Cerenkov angle is dominated by the granularity of the detector.

can be related (16) to the $\mathrm{q} \overline{\mathrm{q}}$ polarized sea distribution through the relation

$$
A_{p}^{K^{-}}=\frac{4 \Delta u^{v}+\Delta d^{v}+\left[(6+4 \eta) \Delta \bar{q}+\left(\frac{\eta}{R}+1\right) \Delta s\right]}{4 u^{v}+d^{v}+\left[(6+4 \eta) \bar{q}+\left(\frac{\eta}{R}+1\right) s\right]}
$$

where $\Delta \overline{\mathrm{q}}$ indicates the polarized sea with $\Delta u_{s}=\Delta \bar{u}_{s}=\Delta d_{s}=\Delta \bar{d}_{s}, \Delta s$ is the polarized strange quark distribution, $u_{v}, d_{v}, \bar{q}$, and $s$ are the unpolarized counterparts. $\eta$ is ratio of favored to unfavored fragmentation functions

$$
\begin{gathered}
D_{\bar{u}}^{K^{-}}(z)=\eta(z) D_{u}^{K^{-}}(z) \\
\eta(z) \approx \frac{1+z}{1-z}
\end{gathered}
$$

In Eq. (15), $R$ gives the suppression of $\bar{u}$ quark fragmentation due to the strange quark mass

$$
D_{\bar{u}}^{K^{-}}(z)=R \cdot D_{s}^{K^{-}}(z) ; \quad R \approx 0.3
$$


Monte Carlo simulations suggest that the statistical precision of a measurement of a $K^{-}$spin asymmetry in HERMES would be sufficient to detect a polarized sea quark distribution provided its polarization is strongly negative, inspite of a substantial dilution of the asymmetry by the valence quark contribution evident in Eq. (15).

\section{ACKNOWLEDGMENTS}

This work is supported by the U.S. Department of Energy, Nuclear Physics Division, under contract W-31-109-ENG-38.

\section{Appendix A. HERMES Collaboration}

P. Green, G. Greeniaus, P. Kitching

University of Alberta, Canada

J.-O. Hansen, H. E. Jackson, C. E. Jones, N. C. R. Makins, T. G. O'Neill, D. Potterveld

Argonne National Laboratory, Argonne, IL USA

E. J. Beise, B. W. Filippone, A. Lung, W. Korsch, R. D. McKeown, M. Pitt W. K. Kellogg Laboratory, Caltech, Pasadena, CA USA

E. Kinney, R. Ristinen, J. Z. Williams

University of Colorado, Boulder, CO USA

W. Beckhusen, B. Grabowski, Y. Holler, K. Sinram, G. Wöbke, K. Zapfe DESY, Hamburg, Germany

H. Böttcher, W.-D. Nowak, H. Roloff, A. Schwind

DESY-IfH, Zeuthen, Germany

M. Düren, F. Neunreither, K. Rith, J. Stenger, F. Stock, W. Wander

University of Erlangen-Nürnberg, Germany

D. H. Beck, R. J. Holt, R. Laszewski, C. N. Papanicolas, S. E. Williamson

University of Illinois, Urbana, IL USA

E. Cisbani, S. Frullani, F. Garibaldi, M. Jodice, G. M. Uriuoli

INFN Sezione Sanita, Rome, Italy

A. Feschenko, V. Lysyakov, I. Savin

JINR, Dubna, Russia

N. Bianchi, G. P. Capitani, V. Muccifora, E. de Sanctis, P. Rossi,

P. Levi Sandri, E. Poli, A. R. Roelon-Cora

LNF, Frascati, Italy

G. R. Court, R. Gamet, P. Hayman, T. Jones, S. Kiourkos

University of Liverpool, England

T. Walcher

University of Mainz, Germany

D. Fick, F. Rathmann

University of Marburg, Germany

R. Ent, J. Kelsey, L. Kramer, K. Lee, R. Milner, R. Redwine, N. Simicevic

Laboratory of Nuclear Science, MIT, Cambridge, MA USA

B. Braun, G. Graw, P. Schiemenz

University of München, Germany

G. Burleson, G. Kyle, B. Park, M.-Z. Wang

New Mexico State University, Las Cruces, NM USA 
J. F. J. van den Brand (Spokesman), M. Doetz, K. de Jager, P. de Witt Huberts

F. Hartjes, B. Kaan, F. Udo

NIKHEF, Amsterdam, The Netherlands

S. Barrow, W. Lorenzon, M. Spangos

University of Pennsylvania, Philadelphia, PA

S. Belostotski, Y. Kiselev, S. Manaenkov, Y. Naryshkin, V. Nelyubin, V. Vikrov

St. Petersburg Nuclear Physics Institute, Catchina

Commonwealth of Independent States

O. Hiroshi, Y. Sakemi, T.-A. Shibata

Tokyo University, Japan

B. Cummings, P. J. Delheij, O. Häusser, R. Henderson, R. Kaiser,

M. Kueckes, C. A. Miller, R. Openshaw, A. Trudel,

M. Vetterli (Deputy Spokesman), R. Woloshyn

Simon Fraser University, TRIUMF, Vancouver, Canada

H. J. Bulten, W. Haeberli, T. Wise, Z. L. Zhou

University of Wisconsin, Madison, WI USA

S. Taroyan, H. Vartapetian, H. Voskanian

Yerevan Physics Institute, Armenia

\section{REFERENCES}

1. Ashman, J., et al. Phys. Lett. B206, 364 (1988); Nucl. Phys. B238, 1 (1989);

Adeva, B. et al., Phys. Lett. B302, 533 (1993).

2. Anthony, P. L., Phys. Lett. 71, 959 (1993); also see Day, D., previous talk, this conference.

3. Jaffee, R. L., and Manohar, A., Nucl. Phys. B337, 509 (1990).

4. Ellis, J., and Jaffee, R. J., Phys. Rev. D9, 1444 (1974).

5. Sokolov, A. A., and Ternov, I. M., Sov. Phys. Doklady 8, 1203 (1964).

6. Frankfurt, L. L., et al., Phys. Lett. B230, 141 (1989).

7. Buon, J., and Steffen, K., Nucl. Instrum. Methods A245, 248 (1986).

8. Bjorken, J. D., Phys. Rev. 148, 1457 (1966); D1, 1367 (1970).

9. Hoodbhoy, P., Jaffe, R. L., and Manohar, A., Nucl. Phys. B312, 571 (1989);

Jaffe, R. L., and Manohar, A., Nucl. Phys. B321, 343 (1989).

10. Jaffe, R. L., and Manohar, Aneesh, Phys. Lett. B223, 218 (1989).

11. Wandzura, W., and Wilczek, F., Phys. Lett. B172, 195 (1977);

Shuriak, E. V., and Vainshtein, A. F., Nucl. Phys. B201, 142 (1982).

12. op cit

13. Mankiewicz, L., Schäfer, A., and Veltri, M., Proceedings of the Workshop "Physics at HERA", Vol. 3, October (1991).

14. Ross, G. G., and Roberts, R. G., "The Glum Contribution to Polarized Nucleon Structure Functions", RAL-90-062 (1990).

15. Schäfer, A., Phys. Lett. B209, 175 (1988).

16. Close, F. E., and Milner, R. G., Phys. Rev. D44, 3691 (1991). 